\title{
Encefalopatía aguda y muerte encefálica en un niño con influenza A (H1N1) 2009 durante la primera ola pandémica
}

\author{
Raúl Bustos B. y Fernando Andrade Y.
}

\section{Acute encephalopathy and brain death in a child with influenza A (H1N1) during the 2009 pandemic}

Since the World Health Organization declared a pandemic of a novel influenza A (H1N1) virus, much attention has been focused on its respiratory manifestations, but limited information regarding neurologic complications has been reported in children. We describe a case of acute encephalopathy progressing to brain death in a pediatric patient with confirmed infection with novel influenza A (H1N1).

Key words: Influenza A (H1N1), pandemic, pediatrics, encephalopathy.

Palabras clave: Influenza A (H1N1), pandemia, pediatría, encefalopatía.

\section{Introducción}

$\mathrm{L}$ a complicaciones neurológicas secundarias a infección por virus influenza -convulsiones febriles, encefalitis, encefalopatía, síndrome de Reye- han sido bien descritas en la literatura ${ }^{1-4}$. La encefalopatía asociada a influenza es una condición rápidamente progresiva que generalmente se presenta en la fase inicial de la enfermedad. Una forma más grave e infrecuente de la encefalopatía asociada a influenza es la llamada encefalopatía necrosante aguda (ENA)-acute necrotizing encephalopathy en la denominación anglosajona- que se acompaña de una mortalidad elevada y secuelas neurológicas graves en los sobrevivientes ${ }^{5}$.

El objetivo de este manuscrito es describir el primer caso en nuestro país de una ENA de evolución rápida a muerte encefálica en un niño con una infección confirmada por el virus influenza A (H1N1) pandémico.

\section{Caso Clínico}

Escolar de 7 años, portador de talasemia menor y antecedentes de convulsión febril a los 3 años de vida. En junio del año 2009, cursó con un cuadro de 24 horas de evolución caracterizado por fiebre de $39^{\circ} \mathrm{C}$, vómitos y diarrea. En su hospital de origen se diagnosticó clínicamente una influenza, por lo que se prescribió oseltamivir $60 \mathrm{mg} / \mathrm{c} 2 \mathrm{~h}(4,8 \mathrm{mg} / \mathrm{kg} /$ día $)$ y se envío a su domicilio donde alcanzó a recibir una dosis del antiviral. En su casa presentó brusca pérdida de conciencia con desviación de la mirada y relajación de esfínter lo que motivó su traslado a nuestra sala de emergencia donde presentó una convulsión tónico-clónica generalizada, que cedió con midazolam ev. Posteriormente, fue ingresado a la unidad de cuidados intensivos con compromiso de conciencia y depresión respiratoria por lo que se intubó y se conectó a ventilación mecánica. No se obtuvieron antecedentes de ingesta de anti inflamatorios no esteroídeos $u$ otro medicamento. Al examen de ingreso destacaban: $\mathrm{T}^{\circ} 39,4$ ${ }^{\circ} \mathrm{C}$, escala neurológica de Glasgow 5, pupilas mióticas e hipertonía generalizada. El examen pulmonar era normal. En los exámenes de laboratorio se observó recuento de leucocitos $9.740 / \mathrm{mm}^{3}$, recuento de plaquetas $221.000 /$ $\mathrm{mm}^{3}$, electrolitos plasmáticos normales, glicemia 128 $\mathrm{mg} / \mathrm{dL}$, PCR $116 \mathrm{mg} / \mathrm{L}$, procalcitonina $12 \mathrm{ng} / \mathrm{ml}$, transaminasas GOT 47 UI, GPT 52 UI, LDH 324UI/L, lactato 2,0 $\mathrm{mmol} / \mathrm{L}$, amonemia $34,4 \mu \mathrm{mol} / \mathrm{L}$, protombinemia $55,4 \%$, TTPA 54,5\%, pH 7,3 bicarbonato $21 \mathrm{mmol} / \mathrm{L}$ y carboxi-hemoglobina $1,3 \%$. Se le realizó una punción lumbar obteniéndose un LCR transparente, incoloro, proteínas $0,19 \mathrm{~g} / \mathrm{L}$, cloruro $7,31 \mathrm{~g} / \mathrm{L}$, glucorraquia 0,85 $\mathrm{g} / \mathrm{L}$ y leucocitos $2 /$ campo (100\% mononucleares). IFI de aspirado nasofaríngeo para los virus influenza, parainfluenza, ADV, y VRS negativo. Reacción de polimerasa en cadena en LCR para VHS negativa. Hemocultivos y cultivo de LCR negativos. Radiografía de tórax normal. Se inició tratamiento empírico con ceftriaxona y aciclovir, manteniéndose el oseltamivir hasta completar 5 días. La TAC cerebral sin contraste sólo reveló hipodensidad focal talámica bilateral; el resto del parénquima cerebral era normal, con surcos corticales conservados (Figuras 1 a y b). El paciente evolucionó en coma persistente y signos clínicos de hipertensión endocraneal iniciándose hiperventilación moderada e infusiones de manitol y sodio hipertónico. A las 9 horas de su ingreso presentó crisis de hipertensión arterial y midriasis bilateral no
Hospital Guillermo Grant Benavente. Concepción, Chile.

Unidad de Cuidados Intensivos Pediátricos (RBB).

Servicio de Imagenología (FAY).

Recibido: 12 de mayo de 2010 Aceptado: 2 de agosto de 2010

Los autores no tienen conflictos de interés con el material publicado.

Correspondencia a: Raúl Bustos Betanzo robustos64@yahoo.com.ar 


\section{Caso Clínico}

Figura 1 a y b. TAC cerebral sin contraste al ingreso: cisternas basales. normales, hipodensidad talámica bilateral, ausencia de edema cerebral.

Figura 2 a y b. TAC cerebral sin contraste efectuada 10 $h$ después del ingreso. Se aprecia colapso de cisternas basales y IV ventrículo, hipodensidad talámica difusa bilateral, edema cerebral difuso. reactiva al estimulo luminoso, asociada a ausencia de reflejos corneales, movimientos espontáneos, flaccidez generalizada y arreflexia, alcanzando escala de Glasgow de 4. Se planteó un enclavamiento de tronco y muerte encefálica. Se le realizó una segunda TAC cerebral que reveló hipodensidad talámica difusa bilateral, edema
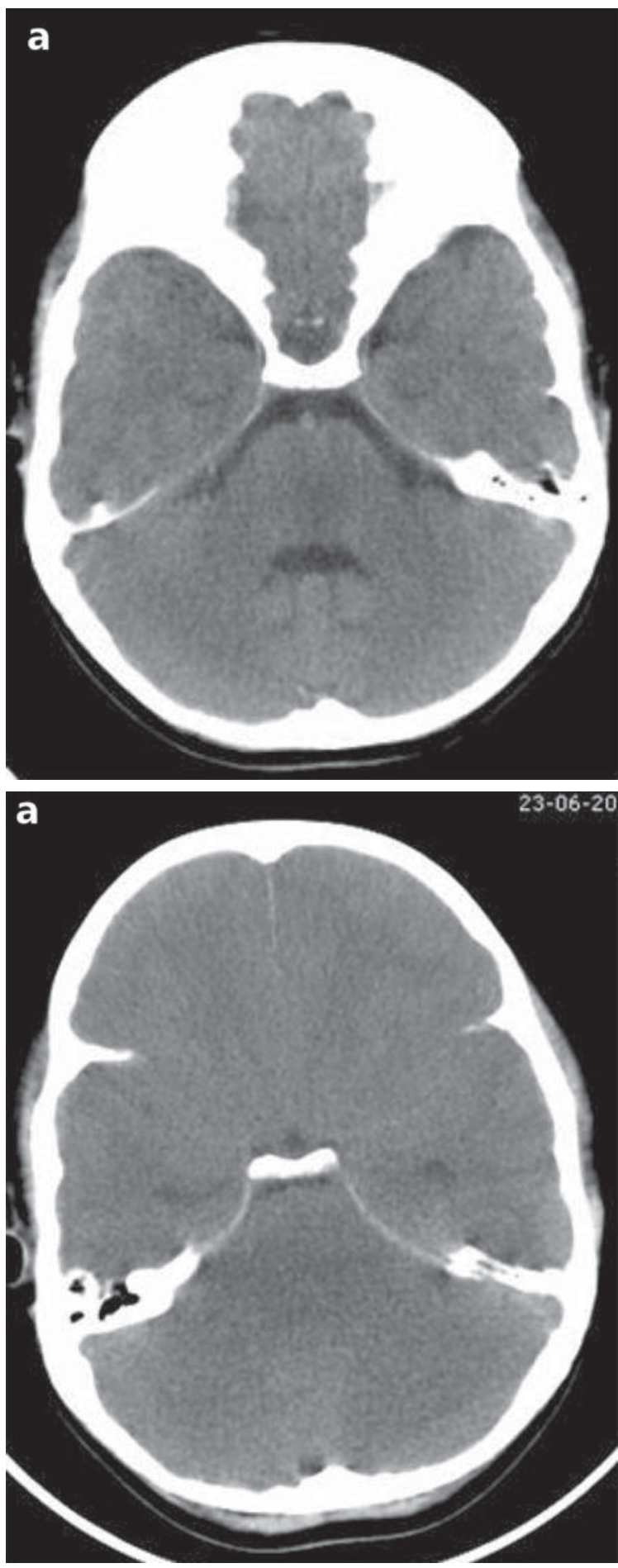

cerebral difuso, colapso de cisternas basales y IV ventrículo (Figuras 2 a y b). La evaluación neuroquirúrgica con miras a monitorizar la presión intracraneal o efectuar una eventual craniectomía descompresiva descartó ambos procedimientos por la condición neurológica del paciente. A las 48 horas del ingreso se repitió la IFI de aspirado
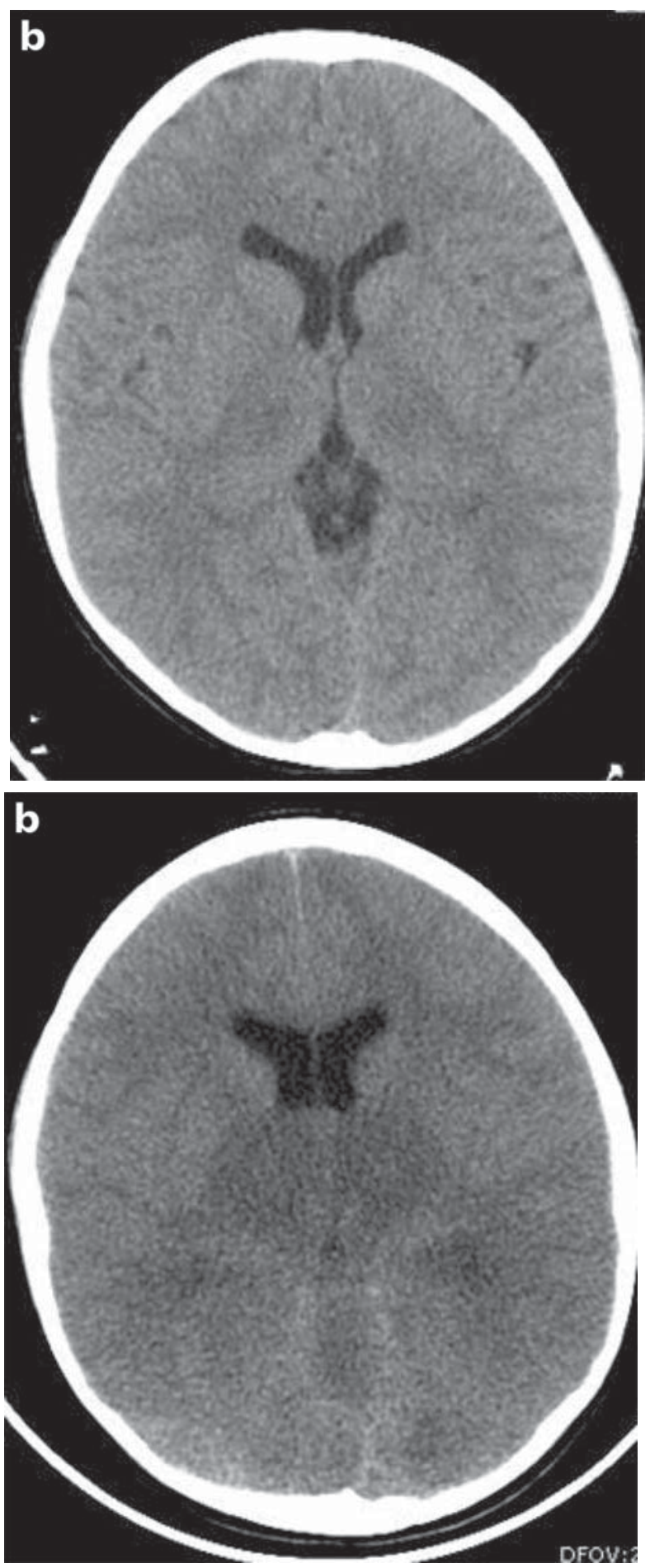
nasofaríngeo la que resultó positiva para virus influenza A(H1N1), hallazgo que fuera posteriormente confirmado por RPC como la cepa pandémica en el Instituto de Salud Pública de Chile, Santiago (laboratorio de referencia nacional). El análisis de laboratorio se realizó utilizando kits para influenza H1N1 pandémica con partidores y sondas específicas para $\mathrm{Sw}$ InfA, Sw H1 pandémica distribuidas por el CDC de Atlanta, EUA, en los centros nacionales de influenza de la red global de la OMS. La RPC para influenza A (H1N1) pandémica en LCR se informó como negativa (Laboratorio Universidad Católica, Santiago). Dos evaluaciones neurológicas y dos EEG realizados con un intervalo de 24 horas fueron compatibles con muerte encefálica. El paciente falleció a los 4 días de su ingreso. Sus padres rechazaron la necropsia.

\section{Discusión}

Durante la pandemia del año 2009, la encefalopatía asociada a influenza $\mathrm{A}(\mathrm{H} 1 \mathrm{~N} 1)$ ha sido reportada en diez niños previamente sanos. La presentación clínica ha incluido compromiso de conciencia, convulsiones, status epiléptico y hemiplejía. Aunque cuatro de estos pacientes son descritos por los autores con secuelas neurológicas menores al alta, en la mayoría de los casos la evolución es benigna ${ }^{6-8}$.

En adultos existen dos series de casos en la literatura, con presentación clínica similar a las series pediátricas y evolución favorable, estudios imagenológicos normales y ausencia de secuelas neurológicas al egreso del hospital $^{9-10}$.

Por el contrario, creemos que el caso aquí presentado, si bien constituye una encefalopatía atribuible a influenza A (H1N1) pandémica, comparte características clínicas, de laboratorio y radiológicas con la ENA, en razón de la rapidez de instalación del coma, convulsiones, ausencia de pleocitosisis en el LCR y asociado a los hallazgos de la TAC: edema y compromiso bilateral de los ganglios basales.

Descrita por primera vez en Japón, la ENA afecta principalmente a niños entre 5 meses y 11 años de edad, alcanzando una mortalidad de $30 \%$ y un handicap neurológico grave en $15 \%$ de los sobrevivientes ${ }^{11}$.

La incidencia de la ENA se desconoce; sin embargo, más de 240 casos han sido reportados en pacientes asiáticos, cinco casos en Norteamérica y diez casos en Europa $^{12}$.

Su presentación clínica es inespecífica pero la combinación de encefalopatía aguda, convulsiones y progresión rápida al coma, más los hallazgos en la TAC de lesiones talámicas bilaterales hacen muy probable el diagnóstico de ENA. Se ha establecido que la ENA se asociada en la mayoría de los casos a infecciones por influenza A(H3N2) y B. También han sido descritos otros agentes causales como VHH6, Coxsackie A9 y Mycoplasma pneumoniae ${ }^{13}$.

En general, la ENA es una condición esporádica y que no recurre. No obstante, casos de ENA familiar y recurrente han sido publicados en relación a mutaciones del gen RANBP2, que codifica una proteína constituyente del poro nuclear en los pacientes afectados y portadores. Recientemente han sido reportados casos que no presentan esta mutación ${ }^{14-17}$.

La patogénesis de la ENA es desconocida. Para algunos autores podría ser consecuencia de una "tormenta" de citoquinas (interleuquina 6 y factor de necrosis tumoral) en respuesta a una infección viral en individuos susceptibles; para otros, el compromiso cerebral seria secundario a un daño vascular cerebral, sin evidencia de penetración del virus a través de la barrera hemato-encefálica, interpretación basada en los hallazgos postmortem consistentes en petequias, congestión y microtrombos de los vasos talámicos. Raramente se ha logrado detectar el genoma del virus influenza en muestras de LCR de los pacientes afectados por lo que un efecto directo se hace poco probable. Otros factores que podrían estar involucrados en la progresión de la enfermedad son una predisposición genética de la población asiática y el uso de medicamentos como diclofenaco sódico, mefenamato y efedrina ${ }^{18}$. Desconocemos si la talasemia menor que presentaba el paciente descrito influyó en su evolución.

El diagnóstico diferencial de la ENA es limitado. El síndrome de Reye y la enfermedad de Leigh, de presentación clínica similar a la ENA, pueden ser excluidas ya que ambas cursan con hiperamonemia, acidosis láctica e hipoglicemia, hallazgos ausentes en nuestro paciente.

Respecto al tratamiento, además de antivirales, en la ENA se han usado pulsos de metilprednisolona, dosis altas de inmunoglobulinas, plasmaféresis e hipotermia, con respuestas disímiles. Es interesante destacar que tres de los pacientes pediátricos con encefalopatía por influenza A (H1N1) pandémica de curso más grave, mencionados en la literatura científica, fueron tratados con dosis altas de metilprednisolona e inmunoglobulinas, observándose en ellos una evolución favorable $\mathrm{e}^{19,20}$.

Actualmente no existe un tratamiento definitivo para la ENA y el manejo consiste en terapia de soporte y de la hipertensión endocraneal, si está presente. Por otro lado, se desconoce la efectividad del tratamiento antiviral en prevenir las complicaciones neurológicas asociadas a influenza A (H1N1) pandémica.

A nuestro entender, este es el tercer caso de encefalopatía aguda por influenza A (H1N1) pandémica, que progresa a muerte encefálica, publicado en la literatura. Lyon describió una niña de 12 años que cursó con encefalopatía y hallazgos en la TAC y RM compatibles con ENA, falleciendo al tercer día de ingresada en muerte cerebral. En la necropsia se observó hemorragias talámica y cerebelosa ${ }^{21}$. El segundo caso es una niña de 7 años que presentó una 
evolución clínica y radiológica prácticamente idéntica a nuestro paciente, con fiebre elevada y compromiso del estado general previo. Se diagnosticó una influenza A por test rápido y fue enviada a su domicilio donde presentó una crisis convulsiva motivando una segunda consulta en emergencia donde se presentó febril y con compromiso de conciencia por lo que requirió intubación y ventilación asistida. Una TAC cerebral reveló edema cerebral difuso, infartos bilaterales de los ganglios basales y borramiento del cuarto ventrículo, falleciendo a los tres días de ser hospitalizada $^{22}$

Finalmente, en un contexto de pandemia, creemos que la influenza por H1N1 debe plantearse como causa de compromiso neurológico en niños, especialmente si cursan con alteración aguda en nivel de conciencia y convulsiones. Un examen de inmunofluorescencia de secreciones respiratorias tiene una baja sensibilidad (cercana a 50\%) para detectar las infecciones por el virus influenza A (H1N1) pandémico, por lo que ante la sospecha debe ser repetido y solicitarse el estudio por RPC.

En conclusión, se comunica el tercer caso pediátrico, comunicado en la literatura médica al momento de enviarse este manuscrito, con características clínicas, de laboratorio e imagenológicas compatible con ENA y atribuible al nuevo virus influenza $\mathrm{A}(\mathrm{H} 1 \mathrm{~N} 1)$ pandémico.

Agradecimientos: Al Instituto de Salud Pública, Departamento de Virología, Sección Virus Respiratorio, a su personal por la confirmación de la existencia del virus pandémico A (H1N1) 2009 en muestra respiratoria.

\section{Resumen}

Desde que la Organización Mundial de la Salud declarara la pandemia por el nuevo virus influenza A (H1N1), gran parte de la atención se ha puesto en sus manifestaciones respiratorias, existiendo escasa información referida a las complicaciones neurológicas de esta condición en niños. Reportamos el curso clínico de un niño con infección confirmada por influenza A (H1N1) pandémico que evolucionó con una encefalopatía aguda y rápida progresión a muerte encefálica.

\section{Referencias}

1.- Morishima T, Togashi T, Yokota S, Okuno Y, Miyakazi C, Masato T, et al. Encephalitis and encephalopathy associated with an influenza epidemic in Japan. Clin Infect Dis 2002; 35: 512-7.

2.- Amin R, Ford-Jones E, Richardson S E, Mac Gregor D, Tellier R, Heurter H, et al. Acute childhood encephalitis and encephalopathy associated with influenza: a prospective 11-year review. Pediatr Infect Dis J 2008; 27: 390-5.

3.- Maricich SM, Neul JL, Lotze TE, Cazacu AC, Uyeki TM, Demmler GJ, et al. Neurologic complications associated with influenza $\mathrm{A}$ in children during the 2003-2004 influenza season in Houston, Texas. Pediatrics 2004; 114: e626-33.

4.- Togashi T, Matsuzono $Y$, Narita M, Morishima T. Influenza associated acute encephalopathy in Japanese children in 19942002. Virus Res 2004; 103: 75-8.

5.- Wang G F, Li W, Li K. Acute encephalopathy and encephalitis caused by influenza virus infection. Curr Opin Neurol 2010; 23: 305-11.

6.- No author listed. Neurologic complications associated with novel influenza A (H1N1) virus infection in children-Dallas, Texas, May 2009. Centers for Disease Control and Prevention (CDC). MMWR Morb Mortal Wkly Rep 2009 24; 58: 773-8.

7.- Baltagi S A, Shoykhet M, Felmet K, Kochanek P M, Bell M J. Neurological sequelae of 2009 influenza A (H1N1) in children: a case series observed during a pandemic. Pediatr Crit Care Med 2010; 11: 179-84.

8.- Webster R I, Hazelton B, Suleiman J, Macartney K, Kesso A, Dale R C. Severe encephalophaty with swine origin influenza A H1N1 infection in childhood: case reports. Neurology 2010; 74: 1077-8.

9.- Noriega L M, Verdugo R J, Araos R, Munita J M, Díaz V, Marcotti A, et al. Pandemic influenza A (H1N1) 2009 with neurological manifestations, a case series. Influenza Other Respi Viruses. 2010; 4: 117-20.

10.- Tan K, Prerna A, Leo Y S. Surveillance of H1N1 related neurological complications Lancet Neurol 2010; 9: 142-3.

11.- Mizuguchi M. Acute necrotizing encephalopathy of childhood: a novel form of acute encephalopathy prevalent in Japan and Taiwan. Brain Dev 1997; 19: 81-92.

12.- Mastroyianni S D, Gionnis D, Vodris K, Skardoutsu A, Mizuguci M. Acute necrotizing encephalophaty of childhood in non Asian patients: report of three cases and literature review. J Child Neurol 2006; 21: 872-9.

13.- Ohsaka M, Houkin K, Takigami M, Koyanagi I. Acute necrotizing encephalopathy associated with human herpesvirus-6 infection. Pediatr Neurol 2006; 34: 160-3.

14.- Marco E J, Anderson J E, Neilson D E, Strober J B. Acute necrotizing encephalopathy in 3 brothers. Pediatrics 2010; 125: e693-8.

15.- Neilson D E, Adams M D, Orr C M, Schelling D K, Eiben R M, Kerr D S, et al. Infection-triggered familial or recurrent cases of acute necrotizing encephalopathy caused by mutations in a component of the nuclear pore, RANBP2. Am J Hum Genet 2009; 84: 44-51.

16.- Gika A D, Rich P, Gupta S, Neilson D E, Clarke A. Recurrent acute necrotizing encephalopathy following influenza $\mathrm{A}$ in a genetically predisposed family. Dev Med Child Neurol 2010; 52: 99-102.

17.- López-Laso E, Mateos-González M E, Pérez-Navero J L, Camino-León R, Briones P, Neilson D E. Infection-triggered familial or recurrent acute necrotizing encephalopathy. An Pediatr (Barc) 2009; 71: 235-9.

18.- Sugaya N. Influenza associated encephalopathy in Japan. Semin Pediatr Infect Dis 2002; 13: 79-84.

19.- Okumura A, Mizuguchi M, Kidokoro H, Tanaa M, Abe S, Hoyosa M, et al. Outcome of acute necrotizing encephalopathy in relation to treatment with corticosteroids and gammaglobulin. Brain Dev 2009; 31 : 221-7.

20.- Munakata M, Kato R, Yokoyama H, Haginoya K, Tanaka Y, Kayaba J, et al. Combined therapy with hypothermia and anticytokine agents in influenza A encephalopathy. Brain Dev 2000; 22: 373-7.

21.- Lyon J B, Remigio C, Milligan T, Deline C. Acute necrotizing encephalopathy in a child with H1N1 influenza infection. Pediatr Radiol 2010; 40: 200-5.

22.- Martin A, Reade E P. Acute necrotizing encephalopathy progressing to brain death in a pediatric patient with novel influenza A (H1N1) infection. Clin Infect Dis 2010; 50: e50-2. 